Article

\title{
Cramer-Rao Lower Bound Evaluation for Linear Frequency Modulation Based Active Radar Networks Operating in a Rice Fading Environment
}

\author{
Chenguang Shi ${ }^{1,2}$, Sana Salous ${ }^{2}$, Fei Wang ${ }^{1}$ and Jianjiang Zhou ${ }^{1, *}$ \\ 1 Key Laboratory of Radar Imaging and Microwave Photonics, Ministry of Education, \\ Nanjing University of Aeronautics and Astronautics, Nanjing 210016, China; scg_space@163.com (C.S.); \\ wangxiaoxian@nuaa.edu.cn (F.W.) \\ 2 School of Engineering and Computing Sciences, Durham University, Durham DH1 3DE, UK; \\ salous.durham@gmail.com \\ * Correspondence: zjjee@nuaa.edu.cn; Tel.: +86-25-8489-2838
}

Academic Editors: Felipe Gonzalez Toro and Antonios Tsourdos Received: 17 September 2016; Accepted: 28 November 2016; Published: 6 December 2016

\begin{abstract}
This paper investigates the joint target parameter (delay and Doppler) estimation performance of linear frequency modulation (LFM)-based radar networks in a Rice fading environment. The active radar networks are composed of multiple radar transmitters and multichannel receivers placed on moving platforms. First, the log-likelihood function of the received signal for a Rician target is derived, where the received signal scattered off the target comprises of dominant scatterer (DS) component and weak isotropic scatterers (WIS) components. Then, the analytically closed-form expressions of the Cramer-Rao lower bounds (CRLBs) on the Cartesian coordinates of target position and velocity are calculated, which can be adopted as a performance metric to access the target parameter estimation accuracy for LFM-based radar network systems in a Rice fading environment. It is found that the cumulative Fisher information matrix (FIM) is a linear combination of both DS component and WIS components, and it also demonstrates that the joint CRLB is a function of signal-to-noise ratio (SNR), target's radar cross section (RCS) and transmitted waveform parameters, as well as the relative geometry between the target and the radar network architectures. Finally, numerical results are provided to indicate that the joint target parameter estimation performance of active radar networks can be significantly improved with the exploitation of DS component.
\end{abstract}

Keywords: Cramer-Rao lower bound (CRLB); Fisher information matrix (FIM); joint parameter estimation; linear frequency modulation (LFM) signal; Rician target; active radar networks

\section{Introduction}

\subsection{Related Works and Motivation}

With widely separated transmitters and receivers, the distributed radar networks, also known as spatial distributed multiple-input multiple-output (MIMO) radar systems [1-3], can view the target from different aspect angles and provide spatial and signal diversities. To be specific, for a distributed radar network system with $M$ transmitters and $N$ receivers, the various transmitter-receiver pairs observe the different aspects of the target. In this way, we can obtain the equivalent of $M N$ radars by optimizing the selection of the transmitted signals from different transmitters. However, the conventional radar observes only single aspect of the target. As we can conclude in [4], the advantage of the radar network is that the average received energy across all the transmitter-receiver pairs is 
approximately constant, and it overcomes deep fades other than the conventional systems. Therefore, the radar network systems have attracted considerable attention and on a path from theory to practice [4-10].

The Cramer-Rao lower bound (CRLB) is an important tool for analyzing the performance of radar networks, which can provide the smallest variance estimates for any unbiased estimation [11,12]. The mean-square error (MSE) of the maximum likelihood estimator (MLE) is close to the CRLB when the high signal-to-noise ratio (SNR) is satisfied. It is also worth mentioning that the performance of multiple signal classification (MUSIC) in computational time-reversal (TR) applications is studied in $[13,14]$, where the closed-form MSE matrix of TR-MUSIC is calculated for the single-frequency case in multistatic co-located and non co-located scenarios. Simulation results show that TR-MUSIC can predict a more accurate MSE than CRLB, while it is a sub-optimal estimator since it does not asymptotically achieve the CRLB as the MLE. In the last couple of years, there is a growing interest on the CRLB studies for the target estimation performance of distributed radar networks [11-19]. The authors in [11] derive the analytical expressions of CRLB for both noncoherent mode and coherent mode in MIMO radar systems, which shows that the CRLB is inversely proportional to the carrier frequency and signals averaged effective bandwidth. In [12], the problem of target parameter estimation for noncoherent MIMO radar is addressed, and the joint CRLB of target position and velocity is computed. Reference [15] further extends the results in [12] to a multiple targets scenario. Later, He et al. investigate the coherent MIMO radar performance when the oscillators at each transmitter and receiver are aligned in phase [16]. The work in [17] studies the target localization accuracy for MIMO radar systems with static phase errors. In [18], the CRLBs of the joint time delay and Doppler shift estimation are derived for an extended target, and the effects of transmitted waveform parameters on the CRLBs are analyzed. Assuming that the approximation state of the target is unknown without previous target detection, a generalized CRLB for distributed active and passive radar networks is calculated in [19].

Recently, the CRLB has been investigated and applied to passive radar systems that employ signals of opportunity as illuminators for target detection, estimation and tracking [20-24]. Since passive radar does not use its own transmitter to radiate electromagnetic wave, it has been a potential technology for low cost, low probability of intercept (LPI) [25-27], antijamming and other advantages. The authors in [20] present the CRLB analysis for the joint target estimation of position and velocity in a frequency modulation (FM) based passive radar networks. In [21-24], the modified CRLB (MCRLB) is employed as a good alternative to the classical CRLB due to the presence of random parameters in the transmitted waveforms, which has been shown to offer a looser bound in practical applications. The target estimation performance of a universal mobile telecommunications systems (UMTS)-based passive multistatic radar and an orthogonal frequency-division multiplexing (OFDM)-based passive radar network in a line-of-sight (LoS) environment is analyzed in $[23,24]$ respectively, where the Rician target model is composed of two components, that is, fixed amplitude or dominant scatterer (DS) and weak isotropic scatterers (WIS). It is shown that the target estimation accuracy will be increased with an increase in reflection coefficient, number of transmitter-receiver pairs, the choice of the transmitter-receiver pairs and duration time. Furthermore, the work in [28] proposes two transmitter of opportunity selection algorithms for FM-based passive radar network systems, which are formulated as knapsack problems (KPs) and tackled with greedy selection approaches. On the basis of the research mentioned above, almost all of previous works focus on stationary platforms. The CRLB analysis for joint moving target position and velocity estimation of linear frequency modulation (LFM) based active radar networks with sensors placed on moving platforms operating in a Rice fading environment, which has not been considered, needs to be investigated.

\subsection{Major Contributions}

The major contributions of this paper are fourfold: 
(1) We formulate the linear frequency modulation (LFM) signal model and derive the log-likelihood function of the received signal for a Rician target. The Rician target model is composed of DS component and WIS components, which are the signals received after striking from the target $[21,22]$. It is worth pointing out here that $[15,16,27]$ only study the target parameter estimation accuracy limits either when the target' radar cross section (RCS) observes as a Rayleigh model in a non-coherent scenario or the target is modeled as a point target in a coherent scenario for all the transmitter-receiver pairs [23]. While utilizing a Rician target model, the estimation performance can be generalized and evaluated when the target has different RCS models for different transmitter-receiver pairs.

(2) On the basis of the previous works [15-24,29], almost all the studies concentrate on stationary platforms. In this paper, we build an LFM-based active radar network configuration and extend it to a more general case, which consist of multiple radar transmitters and multichannel receivers placed on moving platforms. On the other hand, only the CRLBs for LFM-based bistatic radar channels are computed in [29]. To the best of our knowledge, the CRLB for an LFM-based radar network has not been derived. Thus, the joint CRLB for position and velocity estimation of a Rician target in LFM-based radar networks is computed, where we assume that the signals scattered off the target due to different radar transmitters can be received and separated at the receivers. The cumulative Fisher information matrix (FIM) can be factored into two terms: one term accounting for the effect of the DS component, and another incorporating the effect of the WIS components.

(3) Simulation results have shown that the DS component can be exploited to decrease the target parameter estimation errors, which is due to the fact that the reception of DS component increases the received SNR at the radar receiver. Previous results in $[20,29]$ only show that the CRLB is a function of the signal parameters as well as the geometry between the target and the radar network architecture. In this paper, the effects of SNR and target's RCS on the target parameter estimation performance are also analyzed. It is demonstrated that the joint CRLB is not only a function of SNR, target's RCS and transmitted waveform parameters, but also a function of the geometry between the target and the active radar network systems.

(4) The closed-form expressions of CRLB can be used as a performance metric to access the target estimation performance for LFM-based active radar networks in a Rice fading environment. Since the DS component can be exploited to increase the received SNR at the receiver, the geometry-dependent CRLB analysis will open up a new dimension for active radar network systems by aiding the optimal power allocation for radar networks to achieve a given estimation requirement with the minimum system cost.

\subsection{Outline of the Paper}

The rest of the paper is organized as follows. Section 1 describes the signal model for LFM-based radar networks. In Section 2, the joint CRLB is computed for target position and velocity estimation by deriving the closed-form expressions of FIM. The numerical simulations are provided to demonstrate our analytical results in Section 3. Finally, conclusion remarks are drawn with potential future work in Section 4.

Notation: The superscript $T$ represents the transpose operator; $\mathbb{E}\{\cdot\}$ and $(\cdot)^{*}$ represent the expectation and conjugation operators, respectively. $|\cdot|$ denotes the absolute value, $\Re\{\cdot\}$ is the real part, and $\Im\{\cdot\}$ is the imaginary part. $S_{i}(f)$ denotes the Fourier transform of $s_{i}(t)$.

\section{Signal Model}

Consider a active radar network architecture comprising of $N_{T}$ radar transmitters and $N_{R}$ multichannel receivers. Let the $i$ th radar transmitter and the $j$ th receiver be located at $\overrightarrow{\mathbf{p}_{i}^{t}}=\left[x_{i}^{t}, y_{i}^{t}\right]$ and $\overrightarrow{\mathbf{p}_{j}^{r}}=\left[x_{j}^{r}, y_{j}^{r}\right]$ respectively, in a 2-dimensional Cartesian coordinate system for simplicity. The target 
position and velocity are supposed to be deterministic unknown and denoted by $\overrightarrow{\mathbf{p}}=[x, y]$ and $\overrightarrow{\mathbf{v}}=\left[v_{x}, v_{y}\right]$. We define the unknown target state vector:

$$
\mathbf{U}=\left[x, y, v_{x}, v_{y}\right]^{T} .
$$

Without loss of generality, we will concentrate on a single target scenario. However, the results can be extended to multiple targets.

Let $\tau_{i j}$ represent the bistatic time delays corresponding to the path between the $i$ th radar transmitter, moving target, and the $j$ th radar receiver, which is a function of the unknown target position $\overrightarrow{\mathbf{p}}=[x, y]$ :

$$
\begin{aligned}
\tau_{i j} & =\frac{\sqrt{\left(x-x_{i}^{t}\right)^{2}+\left(y-y_{i}^{t}\right)^{2}}+\sqrt{\left(x-x_{j}^{r}\right)^{2}+\left(y-y_{j}^{r}\right)^{2}}}{c} \\
& =\frac{\left\|\overrightarrow{\mathbf{p}}-\overrightarrow{\mathbf{p}_{i}^{t}}\right\|+\left\|\overrightarrow{\mathbf{p}}-\overrightarrow{\mathbf{p}}_{j}^{r}\right\|}{c},
\end{aligned}
$$

where $c$ is the speed of light, $\left\|\overrightarrow{\mathbf{p}}-\overrightarrow{\mathbf{p}_{i}^{t}}\right\|$ denotes the distance from the $i$ th radar transmitter to the target and $\left\|\overrightarrow{\mathbf{p}}-\overrightarrow{\mathbf{p}_{j}^{r}}\right\|$ denotes the distance from the target to the $j$ th receiver, respectively. In this paper, the $i$ th radar transmitter and the $j$ th multichannel receiver are moving with velocities $\overrightarrow{\mathbf{v}}_{i}^{t}=\left[v_{x, i}^{t}, v_{y, i}^{t}\right]$ and $\overrightarrow{\mathbf{v}_{j}^{r}}=\left[v_{x, j}^{r}, v_{y, j}^{r}\right]$, respectively. With the aforementioned positions/velocities of the target, the radar transmitters and receivers, the Doppler shift of the moving target corresponding to the $i j$ th path is the time rate of change of the total $i j$ th path length:

$$
f_{D_{i j}}=\frac{1}{\lambda}\left[\frac{\partial\left\|\overrightarrow{\mathbf{p}}-\overrightarrow{\mathbf{p}_{i}^{t}}\right\|}{\partial t}+\frac{\partial\left\|\overrightarrow{\mathbf{p}}-\overrightarrow{\mathbf{p}_{j}^{r}}\right\|}{\partial t}\right],
$$

where $\lambda$ denotes the carrier wavelength, $\frac{\partial\left\|\overrightarrow{\mathbf{p}}-\overrightarrow{\mathbf{p}_{i}^{t}}\right\|}{\partial t}$ and $\frac{\partial\left\|\overrightarrow{\mathbf{p}}-\overrightarrow{\mathbf{p}_{j}^{f}}\right\|}{\partial t}$ are the relative velocities for the $i$ th radar transmitter and the $j$ th receiver, respectively. Then, we have:

$$
\begin{aligned}
f_{D_{i j}} & =\frac{1}{\lambda}\left[v_{x}\left(\frac{x-x_{i}^{t}}{\left\|\overrightarrow{\mathbf{p}}-\overrightarrow{\mathbf{p}}_{i}^{t}\right\|}+\frac{x-x_{j}^{r}}{\left\|\overrightarrow{\mathbf{p}}-\overrightarrow{\mathbf{p}}_{j}^{t}\right\|}\right)\right]+\frac{1}{\lambda}\left[v_{y}\left(\frac{y-y_{i}^{t}}{\left\|\overrightarrow{\mathbf{p}}-\overrightarrow{\mathbf{p}}_{i}^{t}\right\|}+\frac{y-y_{j}^{r}}{\left\|\overrightarrow{\mathbf{p}}-\overrightarrow{\mathbf{p}}_{j}^{t}\right\|}\right)\right] \\
& +\frac{1}{\lambda}\left[v_{x, i}^{t} \frac{x-x_{i}^{t}}{\left\|\overrightarrow{\mathbf{p}}-\overrightarrow{\mathbf{p}_{i}^{t}}\right\|}+v_{y, i}^{t} \frac{y-y_{i}^{t}}{\left\|\overrightarrow{\mathbf{p}}-\overrightarrow{\mathbf{p}}_{i}^{t}\right\|}\right]+\frac{1}{\lambda}\left[v_{x, j}^{r} \frac{x-x_{j}^{r}}{\left\|\overrightarrow{\mathbf{p}}-\overrightarrow{\mathbf{p}}_{j}^{t}\right\|}+v_{y, j}^{r} \frac{y-y_{j}^{r}}{\left\|\overrightarrow{\mathbf{p}}-\overrightarrow{\mathbf{p}}_{j}^{t}\right\|}\right],
\end{aligned}
$$

which is a function of the unknown target position $\overrightarrow{\mathbf{p}}=[x, y]$ and velocity $\overrightarrow{\mathbf{v}}=\left[v_{x}, v_{y}\right]$.

The LFM signal transmitted by the $i$ th radar transmitter is given by [29]:

$$
s_{i}(t)=\frac{1}{\sqrt{N}} \sum_{n=0}^{N-1} u_{i}\left(t-n T_{R}\right),
$$

where

$$
u_{i}(t)= \begin{cases}\frac{1}{T} e^{j \pi k t^{2}}, & |t| \leq \frac{T}{2} \\ 0, & \text { elsewhere }\end{cases}
$$

$N$ is the number of subpulses for each transmitted burst, $T_{R}$ is the pulse repetition interval (PRI) and $T$ is the duration of each pulse, such that $T<T_{R} / 2$. Moreover, $k T^{2}=B T$ represents the effective time-bandwidth product of the signal and $B$ denotes the total frequency derivation. Note that each 
transmitter-receiver pair has its own angle of view for the target because of the widely spaced antennas that leads to different attenuation factors [20]. It is assumed that the signals from different radar transmitters are supposed to be received and processed at the multichannel radar receivers. For a Rician target, it consists of a DS and many independent WIS. In this paper, by utilizing the Rician target model [23], the reflection coefficient $\zeta_{i j}$ is modeled as a complex Gaussian random variable with mean $d_{i j}$ and variance $\sigma^{2}$, i.e., $\zeta_{i j} \sim \mathcal{C N}\left(d_{i j}, \sigma^{2}\right)$. Then, the signal from the $i$ th radar transmitter arriving at the $j$ th receiver can be expressed as:

$$
r_{i j}(t)=\zeta_{i j} s_{i}\left(t-\tau_{i j}\right) e^{j 2 \pi f_{D_{i j}}\left(t-\tau_{i j}\right)}+w_{i j}(t),
$$

where $w_{i j}(t)$ denotes the additive zero-mean white Gaussian noise of variance corresponding to the $i j$ th path, i.e., $w_{i j} \sim \mathcal{C N}\left(0, \sigma_{n}^{2}\right)$, which is independent to $\zeta_{i j}$. We assume that the parameters $d_{i j}, \sigma^{2}$ and $\sigma_{n}^{2}$ are deterministic and known.

Following the concepts and derivations in [12,23], the likelihood ratio of the $i j$ th transmitter-receiver pair can be given by:

$$
\begin{aligned}
\Lambda\left(r_{i j}(t) ; \mathbf{U}\right) & =\exp \left\{\frac{\sigma^{2}}{\sigma^{2}+\sigma_{n}^{2}}\left|\int_{-\infty}^{+\infty} r_{i j}(t) s_{i}^{*}\left(t-\tau_{i j}\right) e^{-j 2 \pi f_{D_{i j}}\left(t-\tau_{i j}\right)} d t\right|^{2}\right. \\
& -\frac{1}{\sigma^{2}+\sigma_{n}^{2}}\left|\int_{-\infty}^{+\infty} a_{r_{i j}} s_{i}^{*}\left(t-\tau_{i j}\right) e^{-j 2 \pi f_{D_{i j}}\left(t-\tau_{i j}\right)} d t\right|^{2} \\
& +\frac{2}{\sigma^{2}+\sigma_{n}^{2}} \Re\left[\int_{-\infty}^{+\infty} r_{i j}(t) s_{i}^{*}\left(t-\tau_{i j}\right) e^{-j 2 \pi f_{D_{i j}}\left(t-\tau_{i j}\right)} d t\right. \\
& \left.\left.\times d_{r_{i j}}^{*} s_{i}\left(t-\tau_{i j}\right) e^{j 2 \pi f_{D_{i j}}\left(t-\tau_{i j}\right)} d t\right]\right\}+\left(\frac{\sigma_{n}^{2}}{\sigma^{2}+\sigma_{n}^{2}}\right)
\end{aligned}
$$

where $d_{r_{i j}}$ represents the mean of the received signal $r_{i j}(t)$, i.e., $d_{r_{i j}}=d_{i j} s_{i}\left(t-\tau_{i j}\right) e^{j 2 \pi f_{D_{i j}}\left(t-\tau_{i j}\right)}$. Furthermore, the log-likelihood ratio is written as:

$$
\begin{aligned}
L\left(r_{i j}(t) ; \mathbf{U}\right) & =\frac{\sigma^{2}}{\sigma^{2}+\sigma_{n}^{2}}\left|\int_{-\infty}^{+\infty} r_{i j}(t) s_{i}^{*}\left(t-\tau_{i j}\right) e^{-j 2 \pi f_{D_{i j}}\left(t-\tau_{i j}\right)} d t\right|^{2} \\
& -\frac{1}{\sigma^{2}+\sigma_{n}^{2}}\left|\int_{-\infty}^{+\infty} d_{r_{i j}} s_{i}^{*}\left(t-\tau_{i j}\right) e^{-j 2 \pi f_{D_{i j}}\left(t-\tau_{i j}\right)} d t\right|^{2} \\
& +\frac{2}{\sigma^{2}+\sigma_{n}^{2}} \Re\left\{\int_{-\infty}^{+\infty} r_{i j}(t) s_{i}^{*}\left(t-\tau_{i j}\right) e^{-j 2 \pi f_{D_{i j}}\left(t-\tau_{i j}\right)} d t\right. \\
& \left.\times d_{r_{i j}}^{*} s_{i}\left(t-\tau_{i j}\right) e^{j 2 \pi f_{D_{i j}}\left(t-\tau_{i j}\right)} d t\right\}+\ln \left(\frac{\sigma_{n}^{2}}{\sigma^{2}+\sigma_{n}^{2}}\right) .
\end{aligned}
$$

Due to the fact that the radar transmitters and receivers are widely separated, the received signals $r_{i j}(t)$ are mutually independent for different transmitter-receiver pairs. Therefore, the joint log-likelihood ratio across all the transmitter-receiver pairs can be written as the sum of all single transmitter-receiver pair log-likelihood ratios:

$$
\begin{aligned}
L(\mathbf{r}(t) ; \mathbf{U}) & =\sum_{i=1}^{N_{T}} \sum_{j=1}^{N_{R}} L\left(r_{i j}(t) ; \mathbf{U}\right) \\
& =\sum_{i=1}^{N_{T}} \sum_{j=1}^{N_{R}}\left(\Gamma_{i j}^{1}-\Gamma_{i j}^{2}+\Gamma_{i j}^{3}\right)+\mathbb{C},
\end{aligned}
$$

where $\mathbf{r}(t)=\left[r_{11}(t), r_{12}(t), \cdots, r_{N_{T} N_{T}}(t)\right]^{T}$ is the observed signals from the entire set of the receivers. $\Gamma_{i j}^{1}, \Gamma_{i j}^{2}$ and $\Gamma_{i j}^{3}$ denote the first, second and third terms in (9), respectively. The constant 
$\mathbb{C}=\sum_{i=1}^{N_{T}} \sum_{j=1}^{N_{R}} \ln \left(\frac{\sigma_{n}^{2}}{\sigma^{2}+\sigma_{n}^{2}}\right)$ is independent of the target state vector $\mathbf{U}$. Therefore, the MLE of the unknown target state vector $\mathbf{U}$ can be expressed as:

$$
\begin{aligned}
\hat{\mathbf{U}}_{\mathrm{ML}} & =\arg \max _{\mathbf{U}} L(\mathbf{r}(t) ; \mathbf{U}) \\
& =\arg \max _{\mathbf{U}} \sum_{i=1}^{N_{T}} \sum_{j=1}^{N_{R}} L\left(r_{i j}(t) ; \mathbf{U}\right) \\
& =\arg \max _{\mathbf{U}} \sum_{i=1}^{N_{T}} \sum_{j=1}^{N_{R}}\left(\Gamma_{i j}^{1}-\Gamma_{i j}^{2}+\Gamma_{i j}^{3}\right),
\end{aligned}
$$

where $\hat{\mathbf{U}}_{\mathrm{ML}}$ represents the MLE of the unknown parameter vector $\mathbf{U}$.

\section{Derivation of Joint Cramer-Rao Lower Bound}

It is discussed in $[12,16]$ that the CRLB indicates the smallest variance estimate of any unbiased estimate, which can be adopted as a performance metric in parameter estimation problems because that the CRLB is close to the MSE of the MLE when the high SNR is satisfied. Using the derivations in [12,21], the FIM is a $4 \times 4$ matrix related to the second-order derivatives of the joint log-likelihood function:

$$
\begin{aligned}
\mathbf{J}(\mathbf{U}) & =\left(\nabla \mathbf{U} \mathbf{Q}^{T}\right) \mathbf{J}(\mathbf{Q})\left(\nabla \mathbf{U} \mathbf{Q}^{T}\right)^{T} \\
& =\left(\nabla \mathbf{U} \mathbf{Q}^{T}\right)\left(-\mathbb{E}_{\mathbf{r}(t) ; \mathbf{U}}\left\{\nabla \mathbf{Q}[\nabla \mathbf{Q} L(\mathbf{r}(t) ; \mathbf{U})]^{T}\right\}\right)\left(\nabla \mathbf{U} \mathbf{Q}^{T}\right)^{T},
\end{aligned}
$$

where we define $\mathbf{Q}$ as an alternative representation of the unknown parameter vector:

$$
\mathbf{Q}=\left[\tau_{i j}, f_{D_{i j}}\right]^{T}(\forall i, j)
$$

We first derive the Jacobian matrix $\left(\nabla_{\mathbf{U}} \mathbf{Q}^{T}\right)$, whose entries can be obtained by taking the first-order derivatives of the time-delays in (2) and the Doppler shifts in (4) with respect to target positions:

$$
\begin{aligned}
& \frac{\partial \tau_{i j}}{\partial x} \equiv \frac{1}{c}\left(\frac{x-x_{i}^{t}}{\left\|\overrightarrow{\mathbf{p}}-\overrightarrow{\mathbf{p}_{i}^{t}}\right\|}+\frac{x-x_{j}^{r}}{\left\|\overrightarrow{\mathbf{p}}-\overrightarrow{\mathbf{p}_{j}^{r}}\right\|}\right) \\
& \frac{\partial \tau_{i j}}{\partial y} \equiv \frac{1}{c}\left(\frac{y-y_{i}^{t}}{\left\|\overrightarrow{\mathbf{p}}-\overrightarrow{\mathbf{p}_{i}^{t}}\right\|}+\frac{y-y_{j}^{r}}{\left\|\overrightarrow{\mathbf{p}}-\overrightarrow{\mathbf{p}_{j}^{t}}\right\|}\right) \\
& \frac{\partial f_{D_{i j}}}{\partial x} \equiv \frac{1}{\lambda}\left\{v_{x}\left[\frac{\left(y-y_{i}^{t}\right)^{2}}{\left\|\overrightarrow{\mathbf{p}}-\overrightarrow{\mathbf{p}_{i}^{t}}\right\|^{3}}+\frac{\left(y-y_{j}^{r}\right)^{2}}{\left\|\overrightarrow{\mathbf{p}}-\overrightarrow{\mathbf{p}_{j}^{r}}\right\|^{3}}\right]+v_{y}\left[-\frac{\left(x-x_{i}^{t}\right)\left(y-y_{j}^{r}\right)}{\left\|\overrightarrow{\mathbf{p}}-\overrightarrow{\mathbf{p}_{i}^{t}}\right\|^{3}}-\frac{\left(x-x_{j}^{r}\right)\left(y-y_{j}^{r}\right)}{\left\|\overrightarrow{\mathbf{p}}-\overrightarrow{\mathbf{p}_{j}^{r}}\right\|^{3}}\right]\right. \\
& \left.+\left[v_{x, i}^{t} \frac{\left(y-y_{i}^{t}\right)^{2}}{\left\|\overrightarrow{\mathbf{p}}-\overrightarrow{\mathbf{p}_{i}^{t}}\right\|^{3}}-v_{y, i}^{t} \frac{\left(x-x_{i}^{t}\right)\left(y-y_{i}^{t}\right)}{\left\|\overrightarrow{\mathbf{p}}-\overrightarrow{\mathbf{p}_{i}^{t}}\right\|^{3}}\right]+\left[v_{x, j}^{r} \frac{\left(y-y_{j}^{r}\right)^{2}}{\left\|\overrightarrow{\mathbf{p}}-\overrightarrow{\mathbf{p}_{j}^{t}}\right\|^{3}}-v_{y, j}^{r} \frac{\left(x-x_{j}^{r}\right)\left(y-y_{j}^{r}\right)}{\left\|\overrightarrow{\mathbf{p}}-\overrightarrow{\mathbf{p}_{j}^{t}}\right\|^{3}}\right]\right\}, \\
& \frac{\partial f_{i j}}{\partial y} \equiv \frac{1}{\lambda}\left\{v_{x}\left[-\frac{\left(x-x_{i}^{t}\right)\left(y-y_{j}^{r}\right)}{\left\|\overrightarrow{\mathbf{p}}-\overrightarrow{\mathbf{p}_{i}^{t}}\right\|^{3}}-\frac{\left(x-x_{j}^{r}\right)\left(y-y_{j}^{r}\right)}{\left\|\overrightarrow{\mathbf{p}}-\overrightarrow{\mathbf{p}}_{j}^{t}\right\|^{3}}\right]+v_{y}\left[\frac{\left(x-x_{i}^{t}\right)^{2}}{\left\|\overrightarrow{\mathbf{p}}-\overrightarrow{\mathbf{p}_{i}}\right\|^{3}}+\frac{\left(x-x_{j}^{r}\right)^{2}}{\left\|\overrightarrow{\mathbf{p}}-\overrightarrow{\mathbf{p}_{j}^{t}}\right\|^{3}}\right]\right. \\
& \left.+\left[-v_{x, i}^{t} \frac{\left(x-x_{i}^{t}\right)\left(y-y_{i}^{t}\right)}{\left\|\overrightarrow{\mathbf{p}}-\overrightarrow{\mathbf{p}_{i}^{t}}\right\|^{3}}+v_{y, i}^{t} \frac{\left(x-x_{i}^{t}\right)^{2}}{\left\|\overrightarrow{\mathbf{p}}-\overrightarrow{\mathbf{p}}_{i}^{t}\right\|^{3}}\right]+\left[-v_{x, j}^{r} \frac{\left(x-x_{j}^{r}\right)\left(y-y_{j}^{r}\right)}{\left\|\overrightarrow{\mathbf{p}}-\overrightarrow{\mathbf{p}}_{j}^{t}\right\|^{3}}+v_{y, j}^{r} \frac{\left(x-x_{j}^{r}\right)^{2}}{\left\|\overrightarrow{\mathbf{p}}-\overrightarrow{\mathbf{p}_{j}^{*}}\right\|^{3}}\right]\right\},
\end{aligned}
$$

Similarly, the derivatives with respect to the target velocities can be calculated as:

$$
\frac{\partial \tau_{i j}}{\partial v_{x}} \equiv 0,
$$




$$
\begin{gathered}
\frac{\partial \tau_{i j}}{\partial v_{y}} \equiv 0, \\
\frac{\partial f_{D_{i j}}}{\partial v_{x}} \equiv \frac{1}{\lambda}\left(\frac{x-x_{i}^{t}}{\left\|\overrightarrow{\mathbf{p}}-\overrightarrow{\mathbf{p}_{i}^{t}}\right\|}+\frac{x-x_{j}^{r}}{\left\|\overrightarrow{\mathbf{p}}-\overrightarrow{\mathbf{p}_{j}^{r}}\right\|}\right), \\
\frac{\partial f_{D_{i j}}}{\partial v_{y}} \equiv \frac{1}{\lambda}\left(\frac{y-y_{i}^{t}}{\left\|\overrightarrow{\mathbf{p}}-\overrightarrow{\mathbf{p}_{i}^{t}}\right\|}+\frac{y-y_{j}^{r}}{\left\|\overrightarrow{\mathbf{p}}-\overrightarrow{\mathbf{p}_{j}^{r}}\right\|}\right) .
\end{gathered}
$$

After lengthy algebraic derivations, the FIM J $(\mathbf{Q})$ can be correspondingly expressed by:

$$
\begin{aligned}
\mathbf{J}(\mathbf{Q}) & =-\mathbb{E}_{\mathbf{r}(t) ; \mathbf{U}}\left\{[\nabla \mathbf{Q} L(\mathbf{r}(t) ; \mathbf{U})][\nabla \mathbf{Q} L(\mathbf{r}(t) ; \mathbf{U})]^{T}\right\} \\
& =-\mathbb{E}_{\mathbf{r}(t) ; \mathbf{U}}\left\{\nabla \mathbf{Q}[\nabla \mathbf{Q} L(\mathbf{r}(t) ; \mathbf{U})]^{T}\right\} \\
& =\sum_{i=1}^{N_{T}} \sum_{j=1}^{N_{R}} \frac{8 \pi^{2} \sigma^{4}}{\sigma_{n}^{2}\left(\sigma^{2}+\sigma_{n}^{2}\right)}\left[1+2 h_{i j}+\frac{2 h_{i j}}{\left(\sigma^{2} / \sigma_{n}^{2}\right)}\right] \times\left[\begin{array}{cc}
\varepsilon_{i} & \gamma_{i j} \\
\gamma_{i j} & \eta_{i j}
\end{array}\right],
\end{aligned}
$$

where the terms $\varepsilon_{i}, \eta_{i j}$, and $\gamma_{i j}$ are dependent on the radar waveforms, which can be calculated as:

$$
\begin{aligned}
\varepsilon_{i} \equiv \int_{-\infty}^{+\infty} f^{2}\left|S_{i}(f)\right|^{2} d f-\left.\left.\left|\int_{-\infty}^{+\infty} f\right| S_{i}(f)\right|^{2} d f\right|^{2} \\
=\frac{1}{3} \pi^{2} k^{2} T^{2}, \\
\eta_{i j} \equiv \int_{-\infty}^{+\infty} t^{2}\left|s_{i}(t)\right|^{2} d f-\left.\left.\left|\int_{-\infty}^{+\infty} t\right| s_{i}(t)\right|^{2} d f\right|^{2} \\
=\frac{1}{12} T^{2}+\frac{1}{12} T_{R}^{2}\left(N^{2}-1\right), \\
\gamma_{i j} \equiv \Im\left\{\int_{-\infty}^{+\infty} t s_{i}^{*}(t) \frac{\partial s_{i}(t)}{\partial t} d t\right\}-\int_{-\infty}^{+\infty} t\left|s_{i}(t)\right|^{2} d t \int_{-\infty}^{+\infty} s_{i}(t) \frac{\partial s_{i}^{*}(t)}{\partial t} d t \\
=-\frac{1}{6} k \pi T^{2} .
\end{aligned}
$$

The derivation of $\mathbf{J}(\mathbf{Q})$ is provided in Appendix A. Then, we can write the final expression for total FIM across all the transmitter-receiver pairs as:

$$
\mathbf{J}(\mathbf{U})=\sum_{i=1}^{N_{T}} \sum_{j=1}^{N_{R}} \frac{8 \pi^{2} \sigma^{4}}{\sigma_{n}^{2}\left(\sigma^{2}+\sigma_{n}^{2}\right)}\left[1+2 h_{i j}+\frac{2 h_{i j}}{\left(\sigma^{2} / \sigma_{n}^{2}\right)}\right] \mathbf{J}_{i j}(\mathbf{U})
$$

The expressions for the elements of the bistatic FIM $\mathbf{J}_{i j}(\mathbf{U})$ corresponding to the $i j$ th transceiver pair are given in Appendix B. The CRLB for the joint position and velocity estimation of a Rician target can be obtained by taking inverse of FIM in (26), i.e.,

$$
\operatorname{CRLB}(\mathbf{U})=\mathbf{J}^{-1}(\mathbf{U}) \text {. }
$$

Remark 1. It is obvious that the final expression of FIM in (26) is a linear combination of FIMs due to DS component and WIS components [23]. In this paper, one of our goals is to increase the SNR value at the radar receiver by employing the DS component, which leads to lower radar transmit power and better target estimation performance.

Remark 2. From (26) and (27), we can observe that the MCRLB depends on a number of factors. It not only depends on the relative geometry between the target and the radar networks, but also depends on the transmitted LFM waveform parameters such as the duration of each pulse and bandwidth. In addition, it shows dependence on the target's RCS and the SNR. 


\section{Simulation Results}

In the following, numerical results are dedicated to compute the joint CRLB for active active radar networks as well as reveal the effects of several factors on the CRLB.

For numerical simulations, we consider a radar network with five active radar transmitters and an equal number of multichannel receivers, i.e., $N_{T}=5$ and $N_{R}=5$. The Cartesian coordinates of their positions are provided in Figure 1. The position/moving parameters of the radar transmitters are shown in Table 1. The receivers are co-located with the corresponding transmitters and have the same velocities. It is assumed that the target is located at $[6000,6000] \mathrm{m}$ with velocity $[30,50] \mathrm{m} / \mathrm{s}$. For simulation parameters, we set the LFM signal parameters as follows [29]: the number of subpulses $N=256$, the bandwidth $B=50 \mathrm{MHz}$, the duration of each pulse $T=1 \mu \mathrm{s}$, the PRI $T_{R}=0.1 \mathrm{~ms}$, and the carrier wavelength $\lambda=0.03 \mathrm{~m}$.

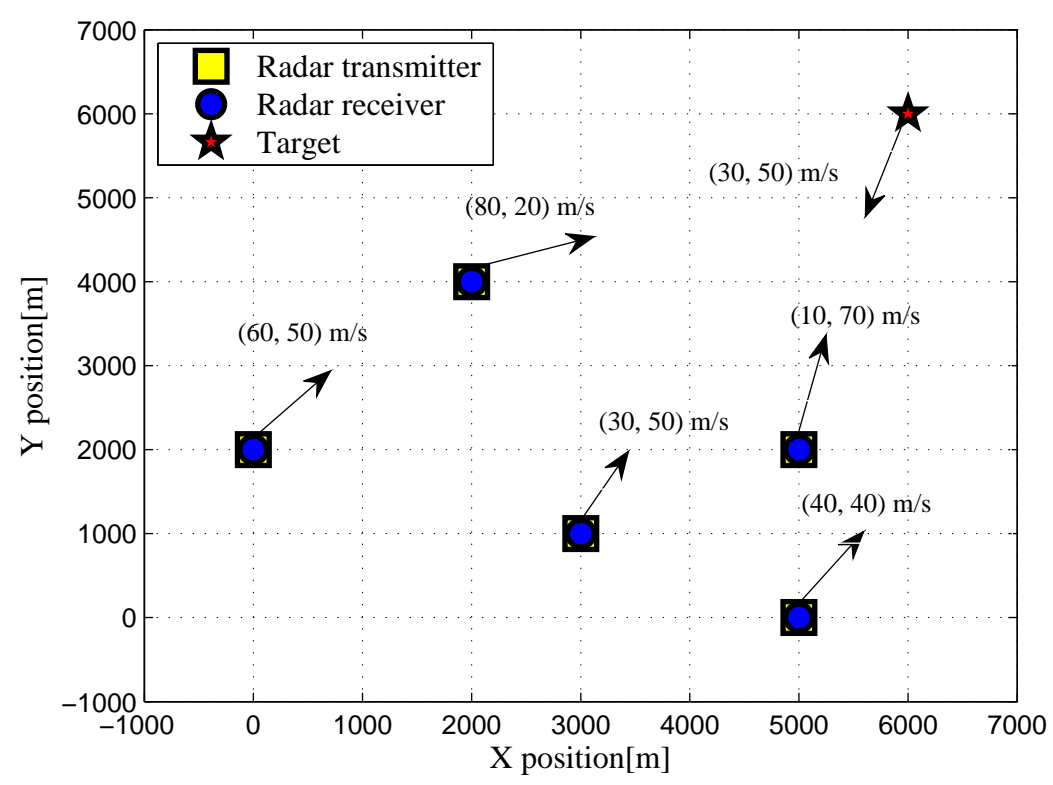

Figure 1. Target and radar networks configuration used in the numerical simulations.

Table 1. Location and Moving Parameters of the Radar Transmitters.

\begin{tabular}{lcc}
\hline Transmitter Index & Locations $[\mathbf{m}]$ & Velocities $[\mathbf{m} / \mathbf{s}]$ \\
\hline Transmitter 1 & {$[3000,1000]$} & {$[30,50]$} \\
Transmitter 2 & {$[5000,2000]$} & {$[10,70]$} \\
Transmitter 3 & {$[2000,4000]$} & {$[80,20]$} \\
Transmitter 4 & {$[0,2000]$} & {$[60,50]$} \\
Transmitter 5 & {$[5000,0]$} & {$[40,40]$} \\
\hline
\end{tabular}

Define the SNR as:

$$
\mathrm{SNR}=10 \log \left(\frac{\sigma^{2}}{\sigma_{n}^{2}}\right)
$$

Without loss of generality, we assume that the reflection coefficients are the same for all transmitter-receiver pairs, i.e., $h_{i j}=h$. In Figures 2 and 3, the MSE curves are plotted versus SNR in the $x$-dimension and y-dimension of target position with different $h$. Solid and dashed curves show the CRLBs and the MSE curves of the ML estimation, respectively. As indicated in [12], it can be observed that the MSE is close to the CRLB in value and slope at an SNR threshold, see the green arrows in the figures. Similarly, we depict the MSE curves of target velocity against SNR in Figures 4 and 5 . 
From Figures 2-5, we can notice that as the value of SNR goes up, the MSE decreases for both target position and velocity estimates.

In addition, it should be pointed out that the CRLB decreases significantly with an increase in $h$. This is due to the fact that an increase in $h$ provides a rise in target RCS [23], which leads to the increase in the received SNR at the radar receiver. The CRLB will achieve a maximum value when DS component does not exist, i.e., $h=0$, where the target RCS follows Rayleigh fluctuations in a non-coherent mode for all the transmitter-receiver pairs. In contrast, the CRLB will be minimum at an asymptotic limit, i.e., $h \rightarrow \infty$, and the target is idealistically a point target in a coherent mode, which has a fixed amplitude RCS value for all the transmitter-receiver pairs. For the rest of the other cases, the CRLB lies in between these two values [23].

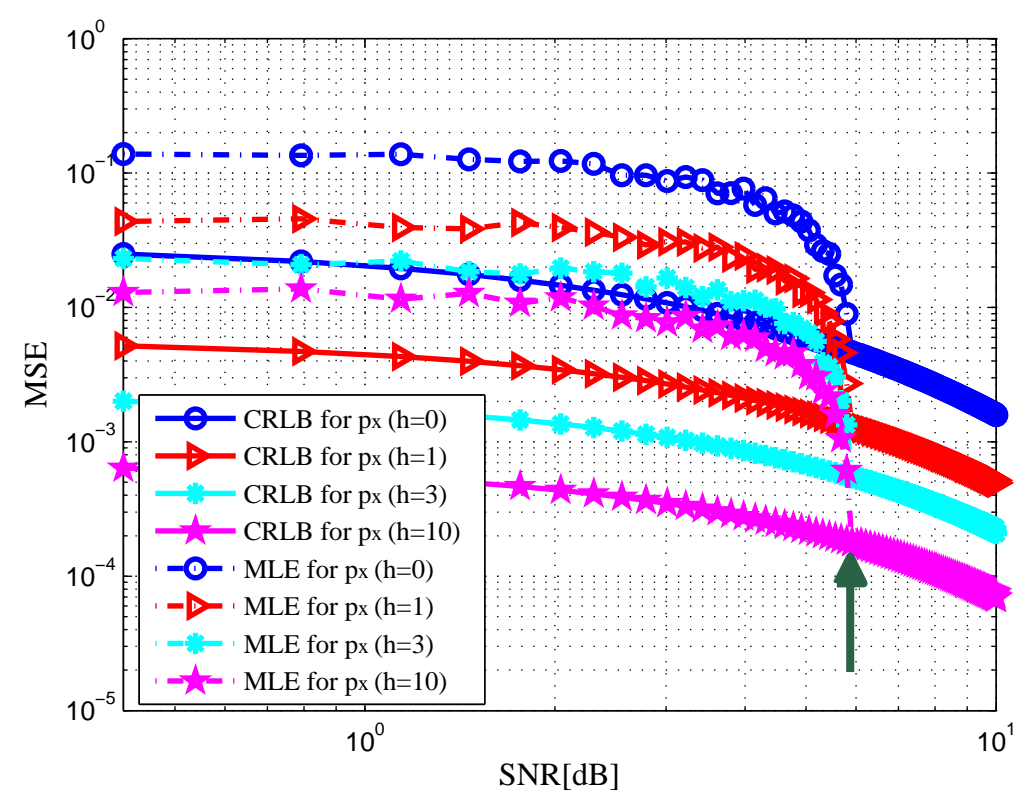

Figure 2. MSE versus SNR for x-dimension of target position with different $h$.

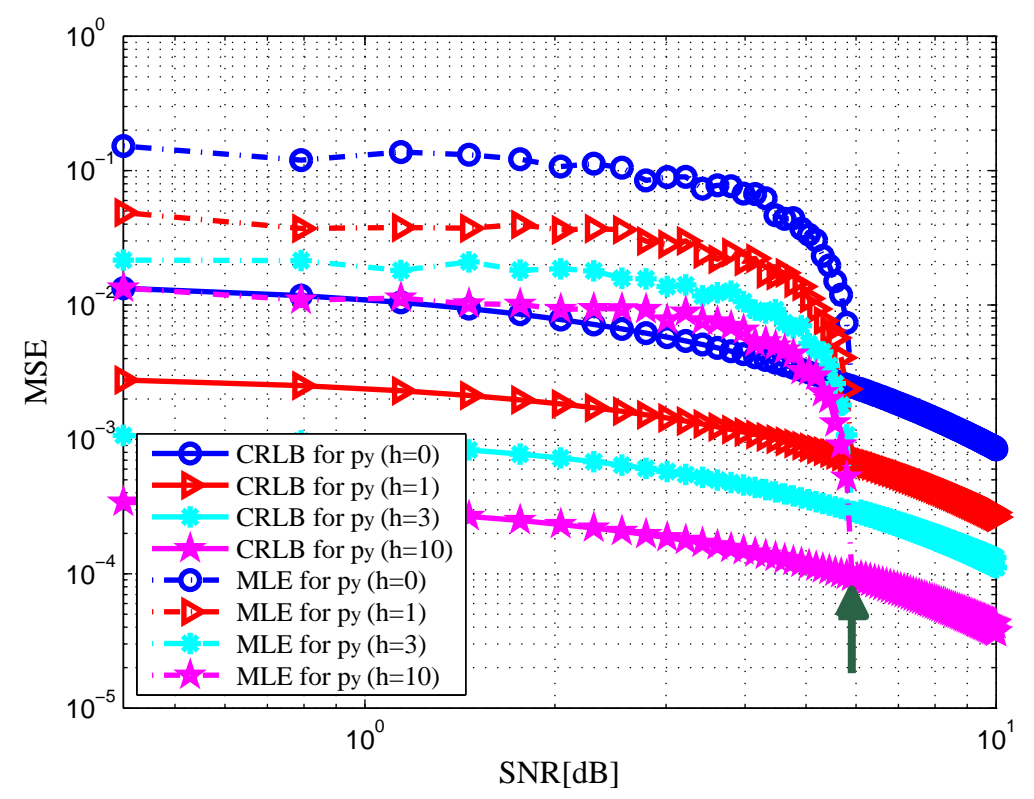

Figure 3. MSE versus SNR for y-dimension of target position with different $h$. 


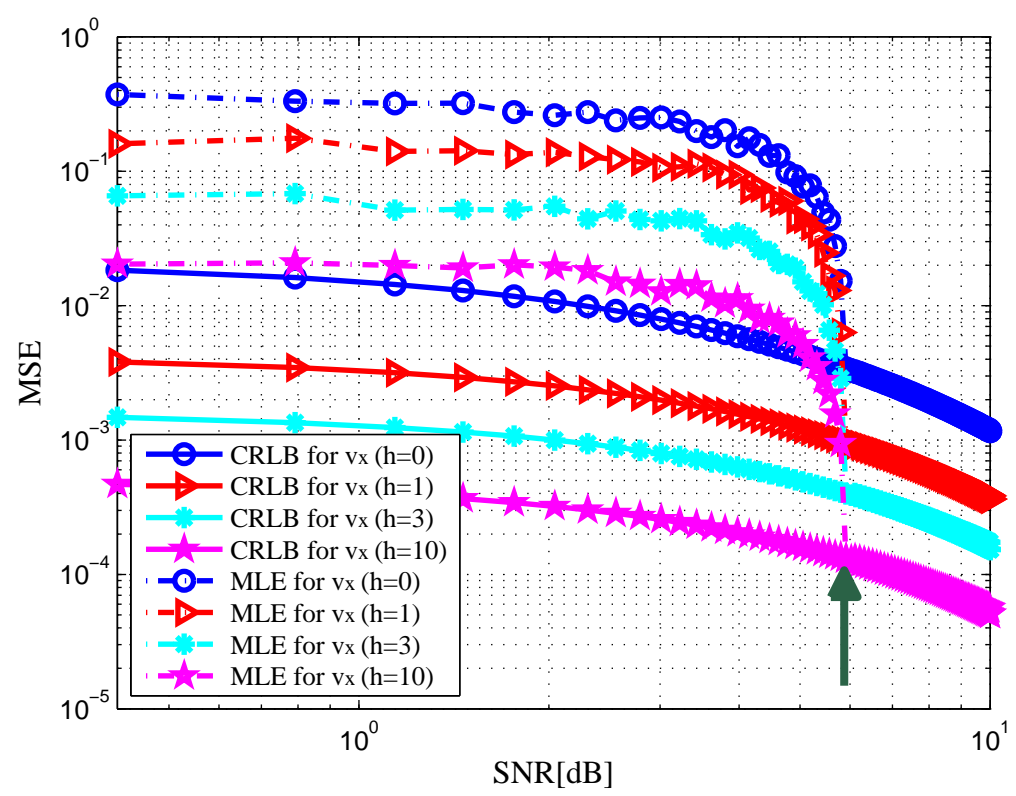

Figure 4. MSE versus SNR for x-dimension of target velocity with different $h$.

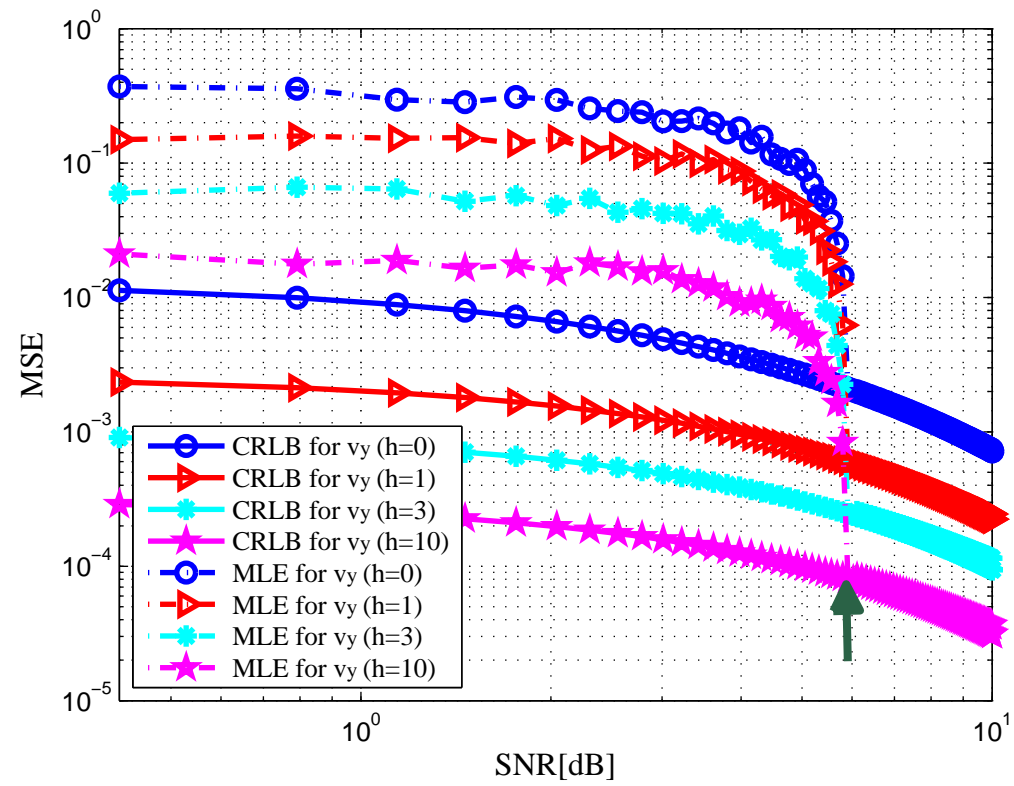

Figure 5. MSE versus SNR for y-dimension of target velocity with different $h$.

Furthermore, we change the location of the target to different positions to investigate the effects of the geometry between the target and the radar networks. In Figures 6-9, we show the CRLBs for both target position and velocity in different position when $\mathrm{SNR}=0 \mathrm{~dB}, h=3$. From these results, we can observe that the CRLBs on the Cartesian coordinates of target position and velocity are different when the target is in different positions. This is because the geometry between the target and the radar network systems impacts the derivatives of the delay-Doppler terms with respect to the Cartesian coordinates significantly $[20,23]$. 


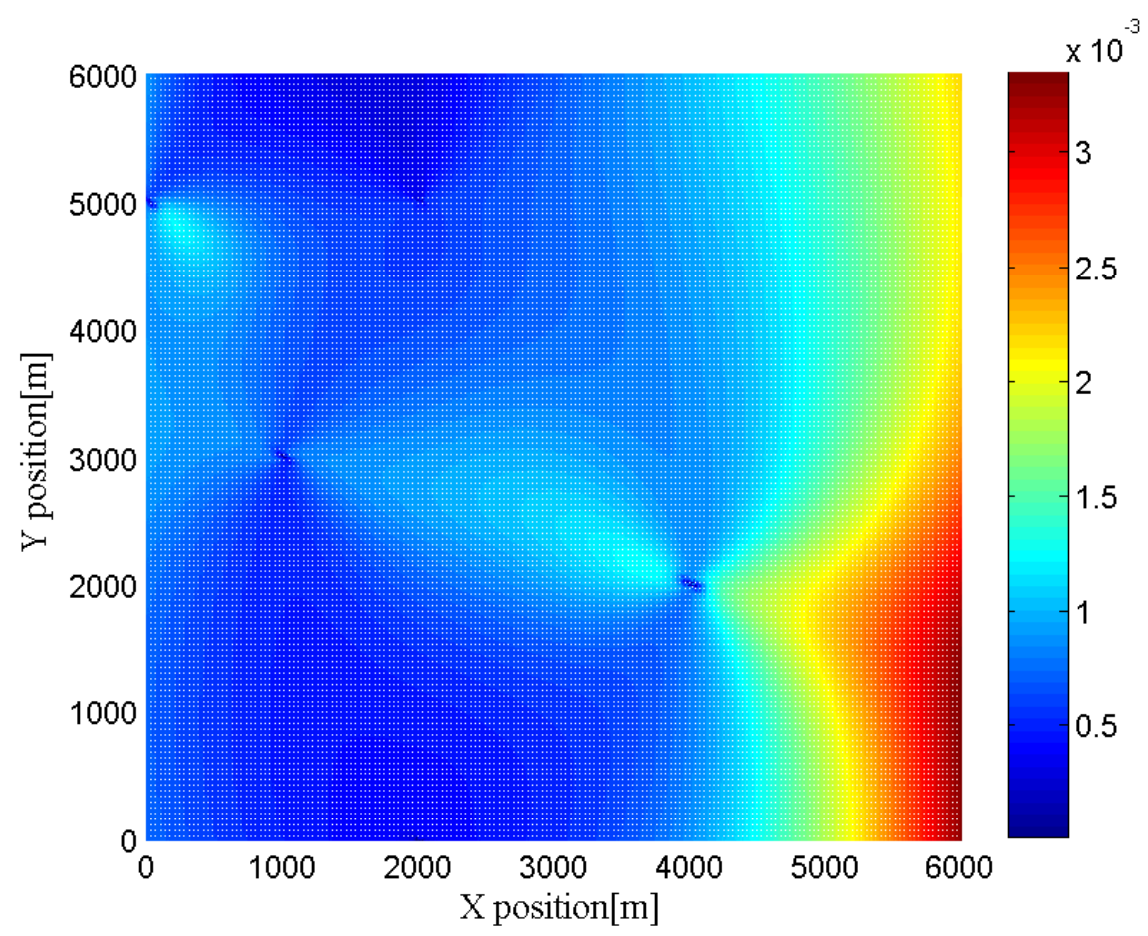

Figure 6. CRLB for $x$-dimension of target position in different position when $\mathrm{SNR}=0 \mathrm{~dB}, h=3$.

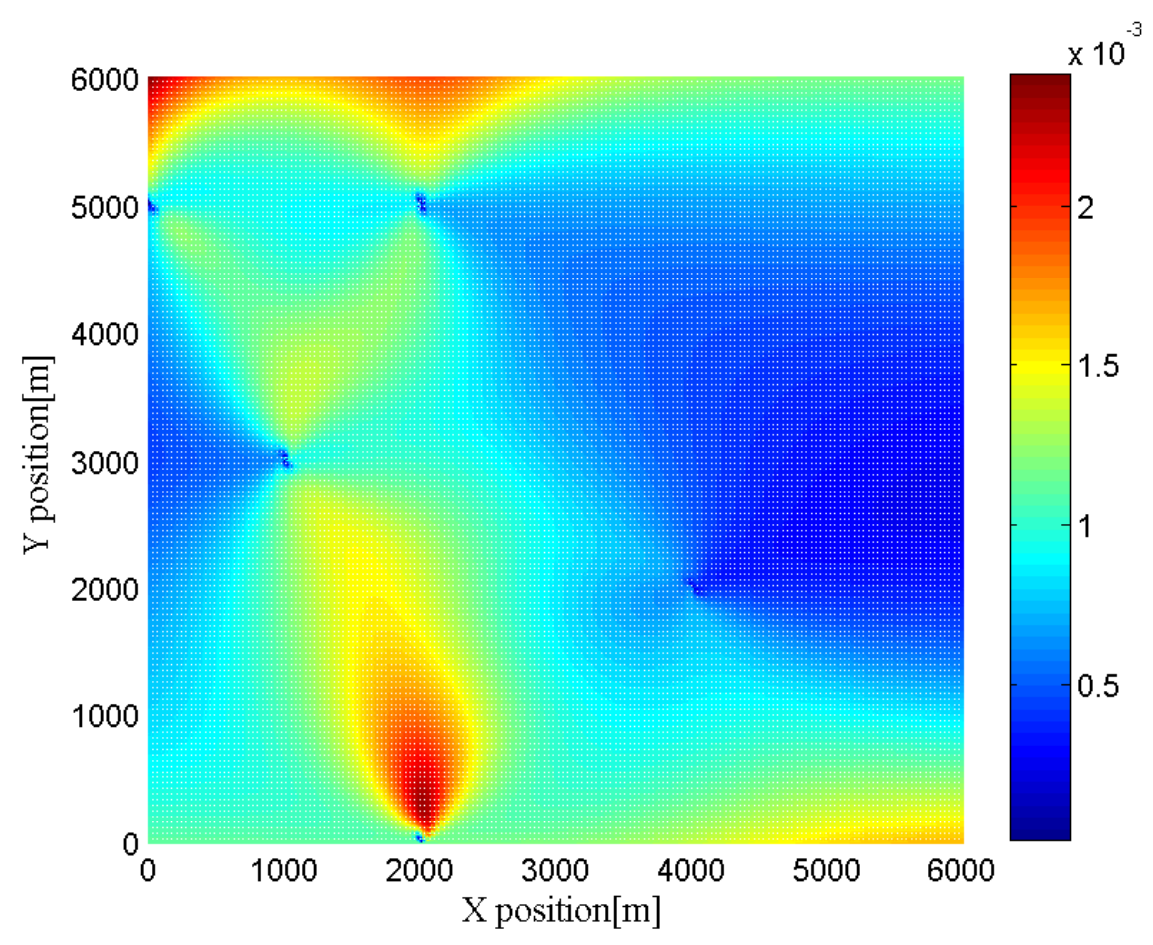

Figure 7. CRLB for $\mathrm{y}$-dimension of target position in different position when $\mathrm{SNR}=0 \mathrm{~dB}, h=3$. 


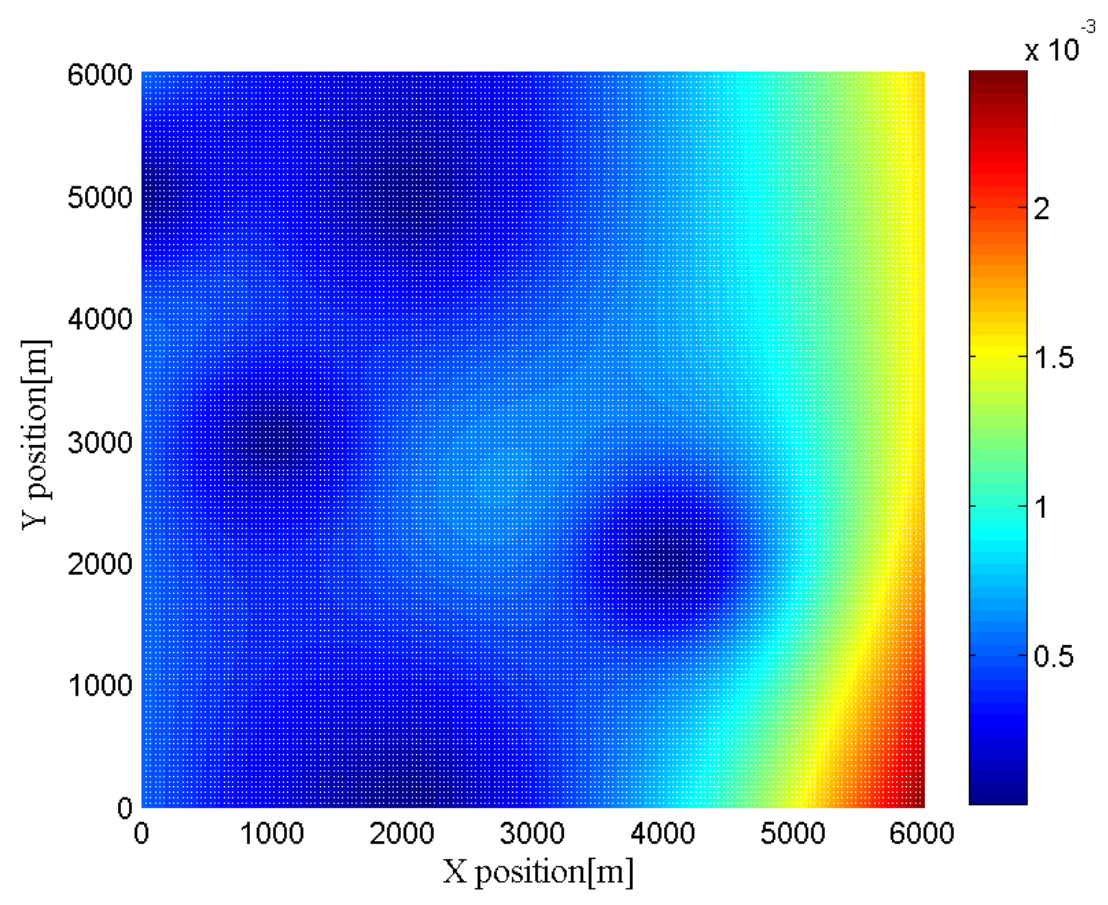

Figure 8. CRLB for $\mathrm{x}$-dimension of target velocity in different position when $\mathrm{SNR}=0 \mathrm{~dB}, h=3$.

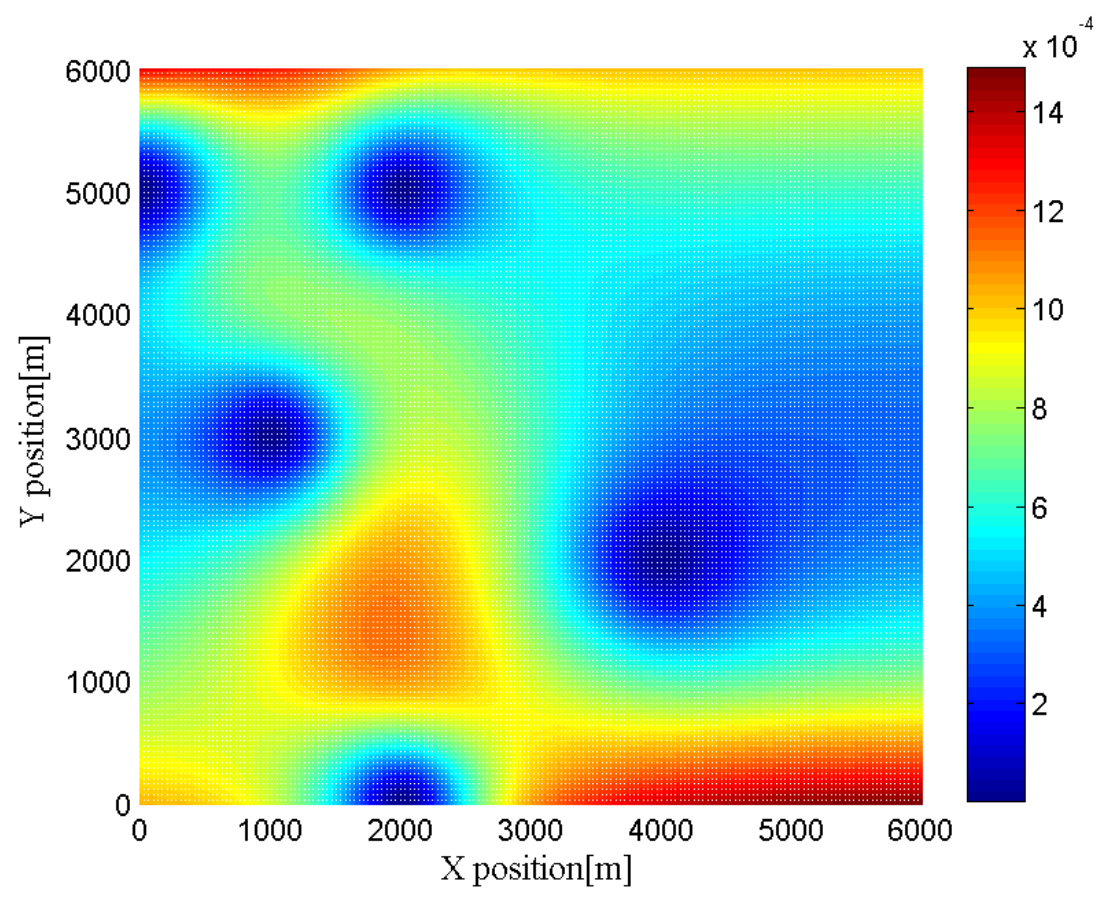

Figure 9. CRLB for $\mathrm{y}$-dimension of target velocity in different position when $\mathrm{SNR}=0 \mathrm{~dB}, h=3$.

In Figure 10, we depict the square root of CRLBs (RCRLBs) for target position coordinates against the duration time of each pulse $T$ and bandwidth $B$ at $0 \mathrm{~dB}$ with different $h$. One can notice that the RCRLBs reduce as the waveform parameters increase, confirming that a waveform with a larger time-bandwidth product can provide better target estimation performance. It is worth mentioning that the figures of target velocity are omitted for the sake of brevity, which are similar to the figures of 
target position. Overall, it can be concluded that the CRLB shows dependence on the SNR, target's RCS, geometry and waveform parameters.

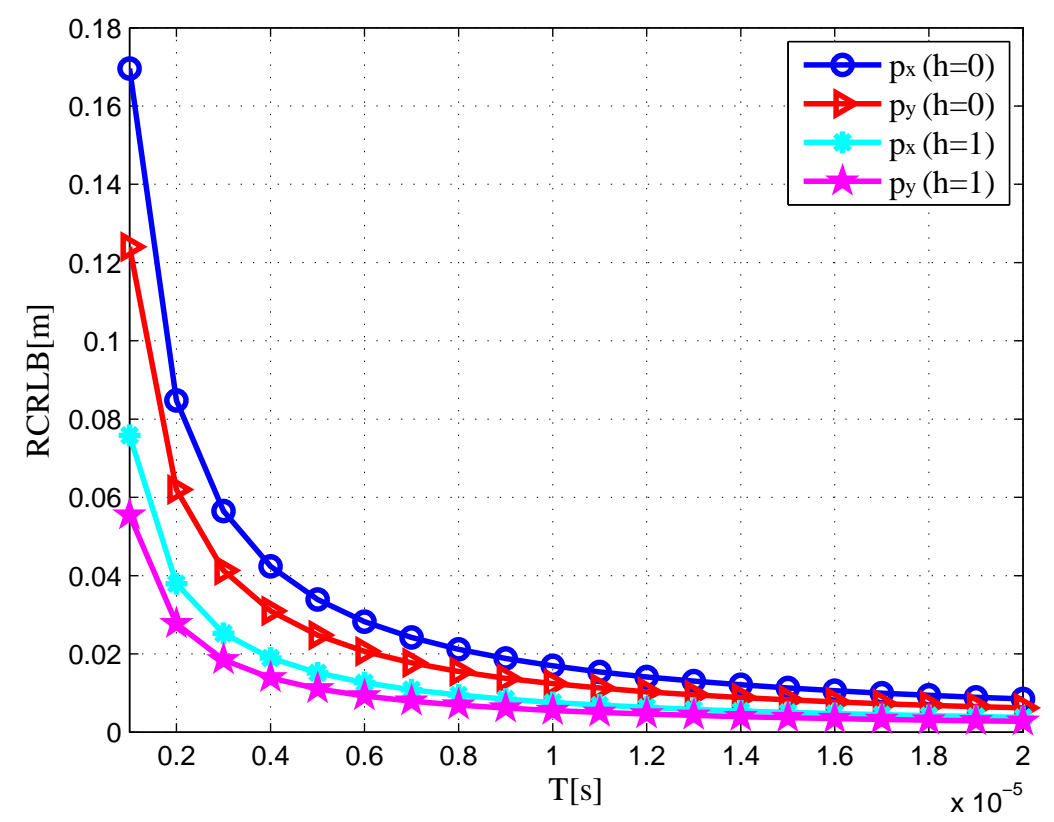

(a)

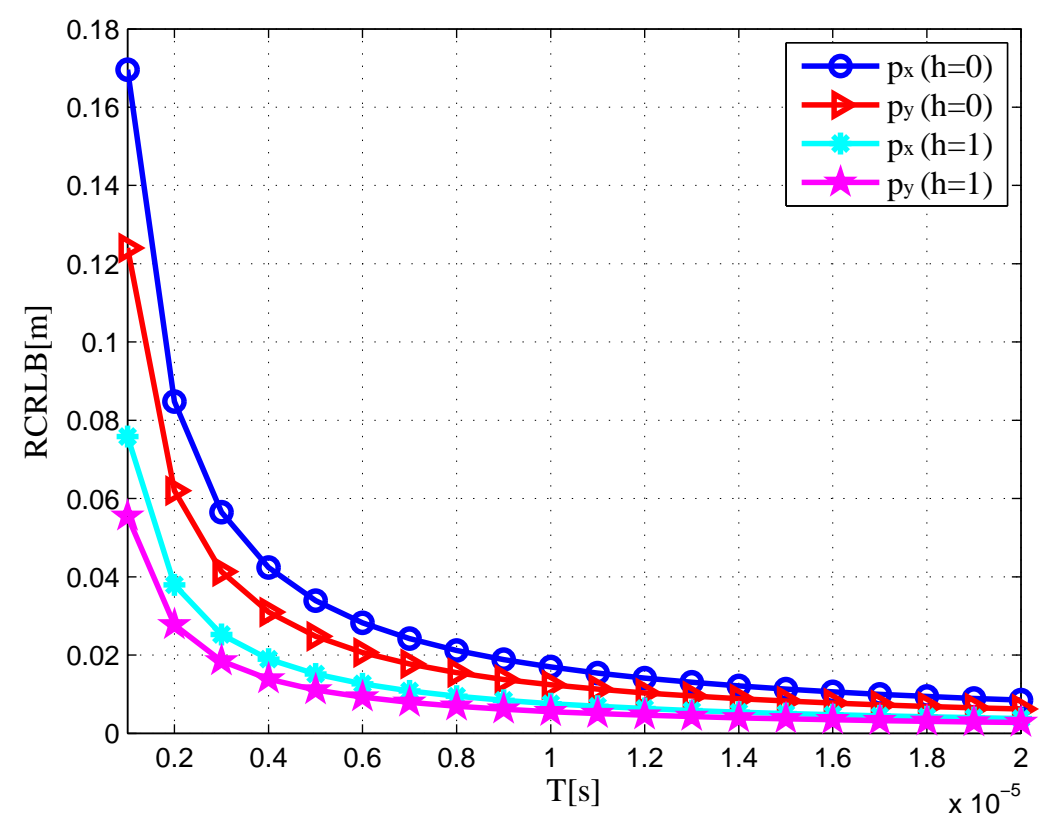

(b)

Figure 10. RCRLB in the target position dimensions versus waveform parameters when $S N R=0 \mathrm{~dB}$ with different $h:(\mathbf{a}) T ;(\mathbf{b}) B$.

\section{Conclusions}

In this paper, we examined the problem of moving target parameter estimation for active radar network systems with sensors on moving platforms in a Rice fading environment, which consist of multiple radar transmitters and multichannel receivers. The CRLB for joint position and velocity estimation of a Rician target has been derived. It should be noted that the cumulative FIM is a linear 
combination of both DS component and WIS components. Numerical examples have been provided to demonstrate that the joint target parameter estimation accuracy of active radar networks can be significantly improved with the exploitation of the DS component. Furthermore, it is shown that the joint CRLB is a function of the transmitted waveforms as well as the geometry between the target and the radar networks. Also, it depends on the SNR and target's RCS. In future work, we will utilize this framework to investigate the problem of optimal power allocation of the radar networks in a Rice fading environment.

Acknowledgments: This research is supported by the National Natural Science Foundation of China (Grant No. 61371170, No. 61671239), the Fundamental Research Funds for the Central Universities (Grant No. NS2015038, No. NP2015404), the National Aerospace Science Foundation of China (Grant No. 20152052028), the Priority Academic Program Development of Jiangsu Higher Education Institutions (PADA) and Key Laboratory of Radar Imaging and Microwave Photonics (Nanjing Univ. Aeronaut. Astronaut.), Ministry of Education, Nanjing University of Aeronautics and Astronautics, Nanjing, 210016, China.

Author Contributions: C.G. Shi, S. Salous and F. Wang conceived and designed the experiments; C.G. Shi, S. Salous and F. Wang performed the experiments; C.G. Shi and J.J. Zhou analyzed the data; C.G. Shi wrote the paper; S. Salous and J.J. Zhou contributed to data analysis revision and English language correction. All authors of article provided substantive comments.

Conflicts of Interest: The authors declare no conflict of interest.

\section{Appendix A. The Derivation of FIM J $(Q)$}

We have:

$$
\Gamma_{i j}^{1}=\frac{\sigma^{2}}{\sigma^{2}+\sigma_{n}^{2}}\left|\int_{-\infty}^{+\infty} r_{i j}(t) s_{i}^{*}\left(t-\tau_{i j}\right) e^{-j 2 \pi f_{D_{i j}}\left(t-\tau_{i j}\right)} d t\right|^{2} .
$$

Let us suppose $\xi_{i j}=\int_{-\infty}^{+\infty} r_{i j}(t) s_{i}^{*}\left(t-\tau_{i j}\right) e^{-j 2 \pi f_{D_{i j}}\left(t-\tau_{i j}\right)} d t$, then the first derivative with respect to $\tau_{i j}$ is given by:

$$
\begin{aligned}
\frac{\partial \Gamma_{i j}^{1}}{\partial \tau_{i j}} & =\frac{\sigma^{2}}{\sigma^{2}+\sigma_{n}^{2}}\left(\xi_{i j}^{*} \frac{\partial \xi_{i j}}{\partial \tau_{i j}}+\xi_{i j} \frac{\partial \xi_{i j}^{*}}{\partial \tau_{i j}}\right) \\
& =\frac{2 \sigma^{2}}{\sigma_{n}^{2}\left(\sigma^{2}+\sigma_{n}^{2}\right)} \times \Re\left(\xi_{i j} \int_{-\infty}^{+\infty} r_{i j}^{*}(t) \frac{\partial s_{i}\left(t-\tau_{i j}\right)}{\partial \tau_{i j}} e^{j 2 \pi f_{D_{i j}}\left(t-\tau_{i j}\right)}\right)
\end{aligned}
$$

To compute the expectation with respect to the second-order derivative, we have:

$$
\begin{aligned}
-\mathbb{E}\left(\frac{\partial^{2} \Gamma_{i j}^{1}}{\partial \tau_{i j}^{2}}\right)= & -\frac{2 \sigma^{2}\left(\sigma^{2}+d_{i j}^{2}\right)}{\sigma^{2}+\sigma_{n}^{2}} \times \mathbb{E}\left[\Re \left(\left|\int_{-\infty}^{+\infty} s_{i}\left(t-\tau_{i j}\right) \frac{\partial s_{i}^{*}\left(t-\tau_{i j}\right)}{\partial \tau_{i j}} d t\right|^{2}\right.\right. \\
& \left.\left.+\int_{-\infty}^{+\infty}\left|s_{i}\left(t-\tau_{i j}\right)\right|^{2} d t \int_{-\infty}^{+\infty} s_{i}^{*}\left(t-\tau_{i j}\right) \frac{\partial^{2} s_{i}\left(t-\tau_{i j}\right)}{\partial \tau_{i j}^{2}} d t\right)\right] .
\end{aligned}
$$

With the derivations in $[29,30]$, hence we obtain:

$$
-\mathbb{E}\left(\frac{\partial^{2} \Gamma_{i j}^{1}}{\partial \tau_{i j}^{2}}\right)=\frac{8 \pi^{2} \sigma^{4}}{\sigma_{n}^{2}\left(\sigma^{2}+\sigma_{n}^{2}\right)}\left(1+2 h_{i j}\right) \varepsilon_{i},
$$

where $h_{i j}=\left|d_{i j}\right|^{2} /\left(2 \sigma^{2}\right)$.

To calculate the expectation with respect to other second-order derivatives, we follow the same procedure and arrive at the following closed form expression:

$$
-\mathbb{E}\left(\frac{\partial^{2} \Gamma_{i j}^{1}}{\partial f_{D_{i j}}^{2}}\right)=\frac{8 \pi^{2} \sigma^{4}}{\sigma_{n}^{2}\left(\sigma^{2}+\sigma_{n}^{2}\right)}\left(1+2 h_{i j}\right) \eta_{i j} .
$$


The off-diagonal terms can be obtained as:

$$
-\mathbb{E}\left(\frac{\partial^{2} \Gamma_{i j}^{1}}{\partial \tau_{i j} \partial f_{D_{i j}}}\right)=\frac{8 \pi^{2} \sigma^{4}}{\sigma_{n}^{2}\left(\sigma^{2}+\sigma_{n}^{2}\right)}\left(1+2 h_{i j}\right) \gamma_{i j} .
$$

Following the same lines, we can get the expectation of second-order derivatives of $\Gamma_{i j}^{2}$ and $\Gamma_{i j}^{3}$ as follows:

$$
\begin{gathered}
-\mathbb{E}\left(\frac{\partial^{2} \Gamma_{i j}^{2}}{\partial \tau_{i j}^{2}}\right)=\frac{8 \pi^{2} \sigma^{4}}{\sigma_{n}^{2}\left(\sigma^{2}+\sigma_{n}^{2}\right)}\left(-\frac{2 h_{i j}}{\sigma^{2} / \sigma_{n}^{2}}\right) \varepsilon_{i}, \\
-\mathbb{E}\left(\frac{\partial^{2} \Gamma_{i j}^{2}}{\partial f_{D_{i j}}^{2}}\right)=\frac{8 \pi^{2} \sigma^{4}}{\sigma_{n}^{2}\left(\sigma^{2}+\sigma_{n}^{2}\right)}\left(-\frac{2 h_{i j}}{\sigma^{2} / \sigma_{n}^{2}}\right) \eta_{i j}, \\
-\mathbb{E}\left(\frac{\partial^{2} \Gamma_{i j}^{2}}{\partial \tau_{i j} \partial f_{D_{i j}}}\right)=\frac{8 \pi^{2} \sigma^{4}}{\sigma_{n}^{2}\left(\sigma^{2}+\sigma_{n}^{2}\right)}\left(-\frac{2 h_{i j}}{\sigma^{2} / \sigma_{n}^{2}}\right) \gamma_{i j}, \\
-\mathbb{E}\left(\frac{\partial^{2} \Gamma_{i j}^{3}}{\partial \tau_{i j}^{2}}\right)=\frac{8 \pi^{2} \sigma^{4}}{\sigma_{n}^{2}\left(\sigma^{2}+\sigma_{n}^{2}\right)}\left(\frac{4 h_{i j}}{\sigma^{2} / \sigma_{n}^{2}}\right) \varepsilon_{i}, \\
-\mathbb{E}\left(\frac{\partial^{2} \Gamma_{i j}^{3}}{\partial f_{D_{i j}}^{2}}\right)=\frac{8 \pi^{2} \sigma^{4}}{\sigma_{n}^{2}\left(\sigma^{2}+\sigma_{n}^{2}\right)}\left(\frac{4 h_{i j}}{\sigma^{2} / \sigma_{n}^{2}}\right) \eta_{i j}, \\
-\mathbb{E}\left(\frac{\partial^{2} \Gamma_{i j}^{3}}{\partial \tau_{i j} \partial f_{D_{i j}}^{3}}\right)=\frac{8 \pi^{2} \sigma^{4}}{\sigma_{n}^{2}\left(\sigma^{2}+\sigma_{n}^{2}\right)}\left(\frac{4 h_{i j}}{\sigma^{2} / \sigma_{n}^{2}}\right) \gamma_{i j} .
\end{gathered}
$$

\section{Appendix B. The Elements of FIM $\mathbf{J}_{i j}(\mathbf{U})$}

The elements of the symmetric FIM $\mathbf{J}_{i j}(\mathbf{U})$ corresponding to the $i j$ th transmitter-receiver pair are given by:

$$
\begin{gathered}
J_{i j}^{11}(\mathbf{U})=\frac{k^{2} \pi^{2} T^{2}}{3}\left(\frac{\partial \tau_{i j}}{\partial x}\right)^{2}-\frac{k \pi T^{2}}{3}\left(\frac{\partial \tau_{i j}}{\partial x}\right)\left(\frac{\partial f_{D_{i j}}}{\partial x}\right)+\frac{1}{12}\left[T^{2}+T_{R}\left(N^{2}-1\right)\right]\left(\frac{\partial f_{D_{i j}}}{\partial x}\right)^{2}, \\
J_{i j}^{12}(\mathbf{U})=J_{i j}^{21}(\mathbf{U})=\left\{\frac{k^{2} \pi^{2} T^{2}}{3}\left(\frac{\partial \tau_{i j}}{\partial x}\right)-\frac{k \pi T^{2}}{6}\left(\frac{\partial f_{D_{i j}}}{\partial x}\right)\right\}\left(\frac{\partial \tau_{i j}}{\partial y}\right) \\
\left.+-\frac{k \pi T^{2}}{6}\left(\frac{\partial \tau_{i j}}{\partial x}\right)+\frac{1}{12}\left[T^{2}+T_{R}\left(N^{2}-1\right)\right]\left(\frac{\partial f_{D_{i j}}}{\partial x}\right)\right\}\left(\frac{\partial f_{D_{i j}}}{\partial y}\right), \\
J_{i j}^{13}(\mathbf{U})=J_{i j}^{31}(\mathbf{U})=\left\{-\frac{k \pi T^{2}}{6}\left(\frac{\partial \tau_{i j}}{\partial x}\right)+\frac{1}{12}\left[T^{2}+T_{R}\left(N^{2}-1\right)\right]\left(\frac{\partial f_{D_{i j}}}{\partial x}\right)\right\}\left(\frac{\partial f_{D_{i j}}}{\partial v_{x}}\right), \\
J_{i j}^{14}(\mathbf{U})=J_{i j}^{41}(\mathbf{U})=\left\{-\frac{k \pi T^{2}}{6}\left(\frac{\partial \tau_{i j}}{\partial x}\right)+\frac{1}{12}\left[T^{2}+T_{R}\left(N^{2}-1\right)\right]\left(\frac{\partial f_{D_{i j}}}{\partial x}\right)\right\}\left(\frac{\partial f_{D_{i j}}}{\partial v_{y}}\right)^{\prime}, \\
J_{i j}^{22}(\mathbf{U})=\frac{k^{2} \pi^{2} T^{2}}{3}\left(\frac{\partial \tau_{i j}}{\partial y}\right)^{2}-\frac{k \pi T^{2}}{3}\left(\frac{\partial \tau_{i j}}{\partial y}\right)\left(\frac{\partial f_{D_{i j}}}{\partial y}\right)+\frac{1}{12}\left[T^{2}+T_{R}\left(N^{2}-1\right)\right]\left(\frac{\partial f_{D_{i j}}}{\partial y}\right)^{2},
\end{gathered}
$$




$$
\begin{gathered}
J_{i j}^{23}(\mathbf{U})=J_{i j}^{32}(\mathbf{U})=\left\{-\frac{k \pi T^{2}}{6}\left(\frac{\partial \tau_{i j}}{\partial y}\right)+\frac{1}{12}\left[T^{2}+T_{R}\left(N^{2}-1\right)\right]\left(\frac{\partial f_{D_{i j}}}{\partial y}\right)\right\}\left(\frac{\partial f_{D_{i j}}}{\partial v_{x}}\right), \\
J_{i j}^{24}(\mathbf{U})=J_{i j}^{42}(\mathbf{U})=\left\{-\frac{k \pi T^{2}}{6}\left(\frac{\partial \tau_{i j}}{\partial y}\right)+\frac{1}{12}\left[T^{2}+T_{R}\left(N^{2}-1\right)\right]\left(\frac{\partial f_{D_{i j}}}{\partial y}\right)\right\}\left(\frac{\partial f_{D_{i j}}}{\partial v_{y}}\right), \\
J_{i j}^{33}(\mathbf{U})=\frac{1}{12}\left[T^{2}+T_{R}\left(N^{2}-1\right)\right]\left(\frac{\partial f_{D_{i j}}}{\partial v_{x}}\right)^{2}, \\
J_{i j}^{34}(\mathbf{U})=J_{i j}^{43}(\mathbf{U})=\frac{1}{12}\left[T^{2}+T_{R}\left(N^{2}-1\right)\right]\left(\frac{\partial f_{D_{i j}}}{\partial v_{x}}\right)^{2}\left(\frac{\partial f_{D_{i j}}}{\partial v_{y}}\right), \\
J_{i j}^{44}(\mathbf{U})=\frac{1}{12}\left[T^{2}+T_{R}\left(N^{2}-1\right)\right]\left(\frac{\partial f_{D_{i j}}}{\partial v_{y}}\right)^{2} .
\end{gathered}
$$

\section{References}

1. Li, J.; Stoica, P. MIMO Radar Signal Processing; Wiley: Hoboken, NJ, USA, 2009; pp. 348-381.

2. Pace, P.E. Detecting and Classifying Low Probability of Intercept Radar; Artech House: Boston, MA, USA, 2009; pp. 319-378.

3. Haimovich, A.M.; Blum, R.S.; Cimini, L.J., Jr. MIMO radar with widely separated antennas. IEEE Signal Process. Mag. 2008, 25, 116-129.

4. Fisher, E.; Haimovich, A.; Blum, R.S.; Cimini, L.J.; Chizhik, D.; Valenzuela, R. Spatial diversity in radars-models and detection performance. IEEE Trans. Signal Process. 2006, 54, 823-836.

5. Naghsh, M.M.; Mahmoud, M.H.; Shahram, S.P.; Soltanalian, M.; Stoica, P. Unified optimization framework for multi-static radar code design using information-theoretic criteria. IEEE Trans. Signal Process. 2013, 61, 5401-5416.

6. Niu, R.X.; Blum, R.S.; Varshney, P.K.; Drozd, A.L. Target localization and tracking in noncoherent multiple-input multiple-output radar systems. IEEE Trans. Aerosp. Electron. Syst. 2010, 48, 1466-1487.

7. Godrich, H.; Tajer, A.; Poor, H.V. Distributed Target Tracking in Multiple Widely Separated Radar Architectures. In Proceedings of the 2012 7th IEEE Sensor Array and Multichannel Signal Processing Workshop (SAM), Hoboken, NJ, USA, 17-20 June 2012; pp. 153-156.

8. Chen, Y.F.; Nijsure, Y.; Yuen, C.; Chew, Y.H.; Ding, Z.G. Adaptive distributed MIMO radar waveform optimization based on mutual information. IEEE Trans. Aerosp. Electron. Syst. 2013, 49, 1374-1385.

9. Godrich, H.; Petropulu, A.P.; Poor, H.V. Sensor selection in distributed multiple-radar architectures for localization: A knapsack problem formulation. IEEE Trans. Signal Process. 2012, 60, 247-259.

10. Song, X.F.; Willett, P.; Zhou, S.L. Optimal Power Allocation for MIMO Radars with Heterogeneous Propagation Losses. In Proceedings of the IEEE International Conference on Acoustics, Speech and Signal Processing (ICASSP), Kyoto, Japan, 25-30 March 2012; pp. 2465-2468.

11. Godrich, H.; Haimovich, A.M.; Blum, R.S. Target localization accuracy gain in MIMO radar-based systems. IEEE Trans. Inf. Theory 2010, 56, 2783-2803.

12. He, Q.; Blum, R.S.; Haimovich, A.M. Noncoherent MIMO radar for location and velocity estimation: More antennas means better performance. IEEE Trans. Signal Process. 2010, 58, 3661-3680.

13. Ciuonzo, D.; Romano, G.; Solimene, R. On MSE Performance of Time-Reversal MUSIC. In Proceedings of the 2014 IEEE 8th Sensor Array and Multichannel Signal Processing Workshop (SAM), A Coruna, Spain, 22-25 June 2014; pp. 13-16.

14. Ciuonzo, D.; Romano, G.; Solimene, R. Performnce analysis of time-reversal MUSIC. IEEE Trans. Signal Process. 2015, 63, 2650-2662. 
15. Wei, C.; He, Q.; Blum, R.S. Cramer-Rao Bound for Joint Location and Velocity Estimation in Multi-Target Non-Coherent MIMO Radars. In Proceedings of the 2010 44th IEEE Annual Conference on Information Sciences and Systems (CISS), Princeton, NJ, USA, 17-19 March 2010; pp. 1-6.

16. He, Q.; Blum, R.S. Noncoherent versus coherent MIMO radar: Performance and simplicity analysis. Signal Process. 2012, 92, 2454-2463.

17. He, Q.; Blum, R.S. Cramer-Rao bound for MIMO radar target localization with phase errors. IEEE Signal Process. Lett. 2010, 17, 83-86.

18. Zhao, T.; Huang, T.Y. Cramer-Rao lower bounds for the joint delay-Doppler estimation of an extended extended target. IEEE Trans. Signal Process. 2016, 64, 1562-1573.

19. Hu, J.B.; He, Q.; Blum, R.S. Comparing the Cramer-Rao Bounds for Distributed Radar with and without Previous Detection Information. In Proceedings of the IEEE China Summit and International Conference on Signal and Information Processing (ChinaSIP), Chengdu, China, 12-15 July 2015; pp. 334-338.

20. Shi, C.G.; Wang, F.; Zhou, J.J. Cramer-Rao bound analysis for joint target position and velocity estimation in FM-based passive radar networks. IET Signal Process. 2016, 10, 780-790.

21. He, Q.; Blum, R.S. The significant gains from optimally processed multiple signals of opportunity and multiple receive stations in passive radar. IEEE Signal Process. Lett. 2014, 21, 180-184.

22. Filip, A.; Shutin, D. Cramer-Rao bounds for L-band digital aeronautical communication system type 1 based passive multiple-input multiple-output radar. IET Radar Sonar Navig. 2016, 10, 348-358.

23. Javed, M.N.; Ali, S.; Hassan, S.A. 3D MCRLB evaluation of a UMTS-based passive multistatic radar operating in a line-of-sight environment. IEEE Trans. Signal Process. 2016, 64, 5131-5144.

24. Shi, C.G.; Salous, S.; Wang, F.; Zhou, J.J. Modified Cramer-Rao lower bounds for joint position and velocity estimation of a Rician target in OFDM-based passive radar networks. Radio Sci. 2016, submitted.

25. Shi, C.G.; Wang, F.; Zhou, J.J.; Zhang, H. Security Information Factor Based Low Probability of Identification in Distributed Multiple-Radar System. In Proceedings of the IEEE International Conference on Acoustics, Speech and Signal Processing (ICASSP), Brisbane, Australia, 19-24 April 2015; pp. 3716-3720.

26. Shi, C.G.; Zhou, J.J.; Wang, F. LPI Based Resource Management for Target Tracking in Distributed Radar Network. In Proceedings of the IEEE Radar Conference (RadarConf), Philadelphia, PA, USA, 2-6 May 2016; pp. 1-5.

27. Shi, C.G.; Wang, F.; Sellathurai, M.; Zhou, J.J.; Zhang, H. Robust transmission waveform design for distributed multiple-radar systems based on low probability of intercept. ETRI J. 2016, 38, 70-80.

28. Shi, C.G.; Wang, F.; Sellathurai, M.; Zhou, J.J. Transmitter subset selection in FM-based passive radar networks for joint target parameter estimation. IEEE Sens. J. 2016, 16, 6043-6052.

29. Greco, M.S.; Stinco, P.; Gini, F.; Farina, A. Cramer-Rao bounds and selection of bistatic channels for multistatic radar systems. IEEE Trans. Aerosp. Electron. Syst. 2011, 47, 2934-2948.

30. Dogandzic, A.; Nehorai, A. Cramer-Rao bounds for estimating range, velocity, and direction with an active array. IEEE Trans. Signal Process. 2001, 49, 1122-1137.

(C) 2016 by the authors; licensee MDPI, Basel, Switzerland. This article is an open access article distributed under the terms and conditions of the Creative Commons Attribution (CC-BY) license (http://creativecommons.org/licenses/by/4.0/). 